\title{
Quasiclassical theory of superconductivity: interfering paths
}

\author{
M. Ozana and A. Shelankov* \\ Department of Theoretical Physics, Umeå University, 90187 Umeå, Sweden
}

(Dated: November 15, 2018)

\begin{abstract}
We apply the method of two-point quasiclassical Green's function to geometries where the trajectories include interfering paths and loops. For a system of two superconducting layers separated by partially transparent interface, corrections to the quasiclassical solutions for the Green's function are explicitly found as well as the deviation from the normalization condition.

PACS numbers: $74.80 \mathrm{Dm}, 74.80 .-\mathrm{g}, 74.50 .+\mathrm{r}, 74.20 . \mathrm{Fg}$
\end{abstract}

The method of quasiclassical Green's function has been long known as the most efficient tool in the theory of superconductivity. In the quasiclassical theory, the superconductor is described by the matrix Green's function which depends on the coordinate and the momentum as well as the energy variable. It obeys the Filenberger equation 1 or its nonstationary generalisation the form of which is similar to the Boltzmann kinetic equation, with the distribution function replaced by a matrix. Following the analogy between the Eilenberger and Boltzmann equations, one may say that the Eilenberger equation describes particles moving along classical trajectories, the matrix nature of the "distribution function" accounting for the internal quantum electron-hole degree of freedom of the quasiparticle in a superconductor. As in the Boltzmann equation case, the notion of a smooth trajectory remains meaningful in the presence of disorder: Although individual impurities may lead to a large angle scattering, the averaging with respect to the impurity positions restores the spatial homogeneity, and the trajectories remain smooth. The presence of disorder (leaving the localization effects out of the picture) is seen only in a finite time that the particle lives on a given trajectory.

In a mesoscopic superconductor, where during its life time the particle may suffer multipe reflections on the boundary, or a bulk sample with interfaces, the typical trajectory may be rather complicated. Compared with classical billiards, trajectories in a superconductor become even more intricate due to the Andreev reflection processes. It has been recently argued in 5 that the derivation of the quasiclassical equation is heavily based on the assumption that the trajectories are single-connected i.e., there exists the unique path which connects any pair of points visited by the particle: The one dimensional character of the trajectory allows one to single out the phase factor due to the motion with the Fermi momentum and derive an equation for a slowly varying envelope, which is the Eilenberger equation. Otherwise, if the particle has alternative paths to go from one point to another, the quantum interference between the paths takes place, which is sensitive to variations of the path on the scale of the Fermi wave length $\lambda_{F}$. In this case, the Fermi wave length oscillations cannot be disentangled from the behaviour at the larger spatial scales of order of the coherence length. Although interfering paths always exist in any realistic situation (e.g. due multiple scattering on impurity), the interference contribution is usually insignificant since it vanishes after averaging with respect to the parameters of the trajectories. This is the reason why the quasiclassical Eilenberger or Boltzmann equations, which completely ignore the interference, work very well.

However, there may be special reasons for the interference to survive the averaging. For instance, the interference of waves propagating along two time reversed path is always constructive if time reversal is a valid symmetry, and it leads to the well-known phenomena of weak localization. The interference may be robust in certain billiard-like geometries where the alternative paths are created by reflections on the boundaries or interfaces. Here, one may invoke the analogy with a classical billiard where intergrability is very sensitive to the geometry. As an example, consider an ideal two-layer system shown in Fig. 1. Trajectories for the case of equal thicknesses of the layers is shown schematically in Fig. 2 (a). For any direction of incidence, the two out-going waves created by the collision with the specular interface combine together after reflection by the outer walls. The trajectory, that is, the set of the points spanned by the particle, is not single-connected. The lengths of the two alternative paths are the same so that their interference is insensitive to the angular averaging and, therefore, may be of importance. (Whether the interference indeed influences observables requires an additional analysis.) The simple of the two recombining paths picture Fig. 2 (a) exist only if iwht a high precision the layers have equal thicknesses, $a^{(l)}=a^{(r)}$. However, the interference is possible for any relation between $a^{(l)}$ and $a^{(r)}$ : For the arbitrary parameter $a^{(l)} / a^{(r)}$, rational or irrational, the wave fragments (partially) recombine after two collisions with the outer walls (see Fig. 2(b)). It is only when the assumption about the specular character of reflection is relaxed, it becomes statistically impossible for the partial waves created by a collision with the interface to meet each other ever again. In these conditions when the alter- 
native paths are absent, the trajectory becomes simply connected, or tree-like in the terminology of 5 . Using the method developed in 5 , one is able to calculate the quasiclassical Green's function on the tree-like trajectory.

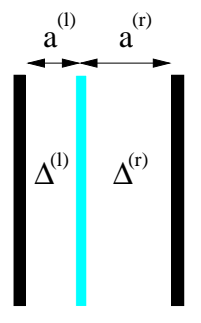

FIG. 1: Sandwich build of two superconductor layers with the order parameters $\Delta^{(l, r)}$ and thicknesses $a^{(l, r)}$ separated by a partially transparent interface.

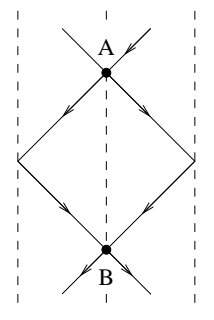

(a)

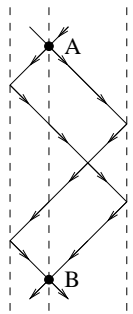

(b)
FIG. 2: Traejectories in a double-layer system with a partially transparent interface. In the ideal system, the reflections are specular. (a) When thicknesses of the layers are equal, the trajectories linked by the collsion with the interface at the point $\mathrm{A}$ meet each other again at the point $\mathrm{B}$ after the specular reflections by the boundaries. (b) For a general irrational relation between the layer thicknesses, the trajectories meet each other meet again after two reflections. The lengths of the two interfering paths connecting the points $\mathrm{A}$ and $\mathrm{B}$ are equal for any direction of the trajectory coming to the point $\mathrm{A}$ so that the interference survives the momentum averaging.

The purpose of this paper is (i) to substantiate the conjecture about the qualitative importance of the single-connectivity of the trajectories in the conventional quasiclassical theory; (ii) to suggest a method which allows one to include the possibility of interfering paths in the quasiclassical theory of superconductivity. Being interested mainly in general questions of the quasiclassical Green's function theory, we consider for definiteness the simplest possible system where the interfering path exist, namely, a sandwich shown in Fig.1. For the specular case, this system is simple enough to solve the full Gor'kov equation and thus to learn "exact" properties. The exact solution being known, one can judge on the validity of approximate schemes and analyse the physical reason for their failure if it happens. It has been demonstrated numerically in 6 that the exact and quasiclassical theory does give a different result in this case. To gain a (a)

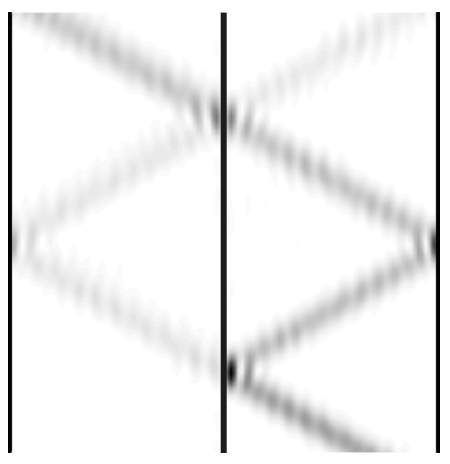

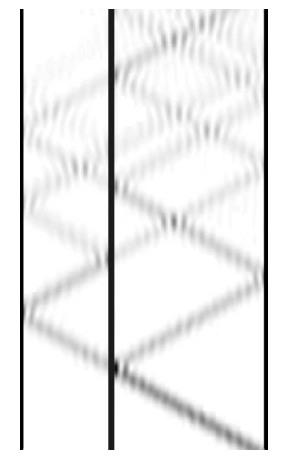

(b)
FIG. 3: Trajectories in a sandwich is half-transparent interface. The intensity is proportional to the logarithm of the absolute value of the exact normal metal Green function after Gaussian average in a small region of the momentum parallel to the interface.

better qualitative understanding of the numerical results of 6 is the main goal of this study.

It is important to realize that the exact solution to the Gor'kov equation must not be directly compared with the quasiclassical solution. For instance, it is clear that the momentum resolved density of states (DOS) found from the full Gor'kov equation for a layer with specular walls, shows $\delta$-function peaks as a function of energy or momentum due to the space quantisation in the layer. On the other hand, the quasiclassical DOS is smooth as in an infinite layer. As discussed ind, it is a coarse-grained Gor'kov Green's function that should be compared with the quasiclassical results. The coarse-grained function is generated by integration of the full Green's function in a small region of $x-p$ space. In particular, one regains a classical picture of trajectories on the coarse-grained level of description. As an illustration of this point, Fig. 3 shows the picture of trajectories produced by the integration of the exact normal metal Green's function $G\left(x, x^{\prime} ; \boldsymbol{p}_{\|}\right) \exp \left[i \boldsymbol{p}_{\|}\left(\boldsymbol{r}-\boldsymbol{r}^{\prime}\right)\right]$ in a small region of $\boldsymbol{p}_{\|}, \boldsymbol{p}_{\|}$ being the in-plane momentum.

The quasiclassical theory of superconductors with interfaces and walls has been discussed in many papers. Surfaces and interfaces violate the condition of applicability of the quasiclassical theory, and their presence is incorporated in the quasiclassical theory through a boundary condition, again similar to the Boltzmann transport equation. For the case of an impenetrable wall, the boundary condition has been derived in Ref.J. The matching condition for a partially transparent specular interface has been derived in Refl 6 as well as 5 . 9 . An isolated interface does not pose severe problems for the quasiclassical theory of superconductivity. The case of two and more interfaces turns out to be more difficult. Analysing the solution to the full Gor'kov equation for a sandwich made of two superconductors with a partially transparent interface, some authors (see 10 , and 11 ) have come to the conclusion that the normalization of 
the Green's function is violated and, therefore, the quasiclassical scheme fails in this case. To a large extent, the present paper has been motivated by the observation made in 11 .

The plan of the paper is the following. In Section we review the method of the 2-point Green's function 12 on an infinite trajectory. In Section III, we derive quasiclassical Green's function for an isolated loop, and discuss the normalisation condition. We show that the normalization condition is violated before the coarse averaging, but not after. In Section III, we consider two coupled loops, and show that the normalisation condition is not valid even on the coarse-grained level. Finally, in Section IV, we apply the results to a superconducting two-layer and analyse applicability of the quasiclassical theory. The physical intepretation of the results is given in Section》.

\section{2-POINT QUASICLASSICAL GREEN'S FUNCTIONS}

We start with a short review of the formulation of the quasiclassical theory in terms of the two-point Green's function on classical trajectories 5 12. Here, we present a novel derivation of the equations in a form convenient for further generalisations.

The main assumption of the quasiclassical theory of superconductivity is the existence of two distinct spatial scales: the short one is the Fermi wave length $\lambda_{F}=1 / p_{F}$, $p_{F}$ being the Fermi momentum, whereas the superconducting coherence length, $\xi_{0}$, exemplifies the large scale. The quasiclassical theory is valid in the zero approximation with respect to the small parameter $\lambda_{F} / \xi_{0} 413$. In effect, the Fermi wave length is put to zero and the orbital motion is essentially classical, that is, the particle coordinate belongs to a chosen classical trajectory. In this picture, the electron-hole mixing, which is the essence of superconductivity, is a quantum internal degree of freedom.

For a given momentum, the direction of velocity is opposite for the electron and hole components of the wave functions, and the derivation should start in a way that both direction on the trajectory enter on equal footing. Consider propagation on a straight line and choose the $x$-axis along the line. Denote the matrix $2 \times 2$ propagator $\mathbb{G}\left(x_{1}, x_{2}\right)$. Throughout the paper, we deal with the retarded Green's function of the Keldysh technique, $\mathbb{G}^{R}$; to simplify notation we omit the superscript $R$. The stationary Green's function satisfies the Gor'kov equation

$$
(\varepsilon-\mathbb{H}) \mathbb{G}\left(x_{1}, x_{2}\right)=\hat{1} \delta\left(x_{1}-x_{2}\right)
$$

where $\varepsilon=\varepsilon+i \delta$ is the energy variable of the retarded Green's function, and the matrix operator $\mathbb{H}$ reads

$$
\mathbb{H}=\left(\begin{array}{cc}
\hat{\xi} & \Delta \\
\Delta^{*} & -\hat{\xi}
\end{array}\right)-\Sigma
$$

where $\Delta(x)$ is the superconducting order parameter, $\Sigma$ is the matrix (retarded) self-energy, and $\xi$ is the kinetic energy,

$$
\hat{\xi}=\frac{\hat{p}_{x}^{2}}{2 m}-\frac{p_{F}^{2}}{2 m} \quad, \quad \hat{p}_{x}=\frac{1}{i} \frac{d}{d x}
$$

with the Fermi energy subtracted. For simplicity, we consider the isotropic case in the absence of a magnetic field. It is straightforward to take into consideration the anisotropy of the order parameter and the vector potential.

The boundary condition to Eq. (1.1) is

$$
\mathbb{G}\left(x_{1}, x_{2}\right) \rightarrow 0 \quad, \quad\left|x_{1}-x_{2}\right| \rightarrow \infty
$$

as it follows from the requirement of the analyticity of the Green's function.

Considered as a function of the first argument $x_{1}$, the Green's function $\mathbb{G}$ in Eq. (1.1) describes the wave propagating from the point $x_{2}$. If the energy variable $\varepsilon$ is small in comparison with the Fermi energy $p_{F}^{2} / 2 m$, the wave has two components with the momenta close to $+p_{F}$ or $-p_{F}$. In the quasiclassical theory, these components are ascribed to two trajectories with the opposite momentum, and the corresponding trajectory propagators obey separate inhomogeneous equations. To carry out the separation, we use the following procedure.

The $\delta$-function inhomogeneity in Eq. (1.1), that is the source of the wave, is equivalent to the following conditions at the points $x_{1}=x_{2} \pm 0$

$$
\begin{aligned}
\left.\mathbb{G}\left(x_{1}, x_{2}\right)\right|_{x_{1}=x_{2}-0} ^{x_{1}=x_{2}+0} & =0 \\
\left.\frac{1}{2 m} \frac{d}{d x_{1}} \mathbb{G}\left(x_{1}, x_{2}\right)\right|_{x_{1}=x_{2}-0} ^{x_{1}=x_{2}+0} & =\tau_{z} .
\end{aligned}
$$

Defining the differential operators $D_{x}^{ \pm}$

$$
D_{x}^{ \pm}=\frac{1}{2} \pm \frac{1}{2 i p_{F}} \frac{d}{d x},
$$

one rewrites Eqs. (1.4) and (1.5), as the following two inhomogeneous equations,

$$
\left.i v_{F} D_{x_{1}}^{ \pm} \mathbb{G}\left(x_{1}, x_{2}\right)\right|_{x_{1}=x_{2}-0} ^{x_{1}=x_{2}+0}=\hat{1}
$$

These conditions can be satisfied by the following decomposition:

$$
\mathbb{G}\left(x_{1}, x_{2}\right)=\sum_{\sigma= \pm} \mathbb{G}^{\sigma}\left(x_{1}, x_{2}\right),
$$

where the partial propagators $\mathbb{G}^{ \pm}$obey the homogeneous linear equation Eq. (1.1) at $x_{1} \neq x_{2}$ and the inhomogeneous conditions

$$
\left.i v_{F} D_{x_{1}}^{\sigma^{\prime}} \mathbb{G}^{\sigma}\left(x_{1}, x_{2}\right) \tau_{z}\right|_{x_{1}=x_{2}-\sigma \cdot 0} ^{x_{1}=x_{2}+\sigma \cdot 0}=\delta_{\sigma \sigma^{\prime}} \hat{1} \quad, \quad \sigma, \sigma^{\prime}= \pm
$$

which replace the $\delta$-function source in the r.h.s. of Eq. (1.1). 
Now, one observes that $\mathbb{G}^{+}$and $\mathbb{G}^{-}$correspond to the opposite direction of the Fermi momentum i.e. they can be presented as

$$
i v_{F} \mathbb{G}^{ \pm}\left(x_{1}, x_{2}\right) \tau_{z}=g^{ \pm}\left(x_{1}, x_{2}\right) e^{ \pm i p_{F}\left(x_{1}-x_{2}\right)},
$$

where $g^{ \pm}\left(x_{1}, x_{2}\right)$ is a slowly varying function if the energy $\varepsilon$ is small in comparison with the Fermi energy; the factor with the Fermi velocity $v_{F}=p_{F} / m$ and the Pauli matrix $\tau_{z}$ are introduced for future convenience.

Indeed, for slowly varying $g^{ \pm}$,

$\frac{1}{i} \frac{d}{d x_{1}} g^{ \pm}\left(x_{1}, x_{2}\right) e^{ \pm i p_{F}\left(x_{1}-x_{2}\right)} \approx \pm p_{F} g^{ \pm}\left(x_{1}, x_{2}\right) e^{ \pm i p_{F}\left(x_{1}-x_{2}\right)}$

Assuming that typically $g^{ \pm}\left(x_{1}, x_{2}\right)$ vary on the coherence length $\xi_{0}$, the corrections to this relation are of order of $\lambda_{F} / \xi_{0}$. Small quantities of this order are consistently neglected in the quasiclassical theory. Note that this formula correspond to the Andreev approximation 14 where the derivatives of quasiclassical envelope functions are always neglected if they stand next to $p_{F}$.

With the accuracy Eq. (1.9) holds, $D_{x}^{ \pm}$are projectors, i.e.,

$$
D_{x_{1}}^{\sigma} \sum_{\sigma^{\prime}= \pm} \mathbb{G}^{\sigma^{\prime}}\left(x_{1}, x_{2}\right)=\mathbb{G}^{\sigma}\left(x_{1}, x_{2}\right)
$$

The projecting property guarantees that one of the conditions in Eq. (1.7), namely, corresponding to $\sigma^{\prime}=-\sigma$, is automatically satisfied. The remaining conditions read

$$
\begin{gathered}
\left.g^{+}\left(x_{1}, x_{2}\right)\right|_{x_{1}=x_{2}-0} ^{x_{1}=x_{2}+0}=\hat{1}, \\
\left.g^{-}\left(x_{1}, x_{2}\right)\right|_{x_{1}=x_{2}+0} ^{x_{1}=x_{2}-0}=\hat{1} .
\end{gathered}
$$

It is clear that $g^{+}$and $g^{-}$are associated with the Fermi surface points $+p_{F} \boldsymbol{n}$ and $-p_{F} \boldsymbol{n}$, respectively, $\boldsymbol{n}$ being the orientation of the $x$-axis. Spanning different orientation . of the $x$-axis, one defines function $g\left(x_{1}, x_{2} \mid \boldsymbol{n}, \boldsymbol{R}\right)$ where $\boldsymbol{n}$ and $\boldsymbol{R}$ are the parameters of the trajectory, and $x_{1,2}$ are the coordinates on the trajectory see Fig. 4 . Then information on the direction of the momentum is contained in $\boldsymbol{n}$, and the superscript \pm in $g^{ \pm}$, being redundant, can be omitted.

Plugging the ansatz Eq. (1.8) into Eq. (1.1), and using the Andreev approximation

$$
e^{-i p_{F} x} \hat{\xi} e^{i p_{F} x} \approx-i v_{F} \frac{\partial}{\partial x}
$$

one obtains the first order differential equation for $g$.

The 2-point Green's function $\hat{g}_{\varepsilon}\left(x_{1}, x_{2} \mid \boldsymbol{n}, \boldsymbol{R}\right)$ obeys the following set of equation 12 :

$$
\begin{aligned}
\left(i v_{F} \frac{\partial}{\partial x_{1}}-\Phi\left(\boldsymbol{r}_{1}\right)+\hat{H}_{\varepsilon, \boldsymbol{n}}\left(\boldsymbol{r}_{1}\right)\right) \hat{g}_{\varepsilon}\left(x_{1}, x_{2} \mid \boldsymbol{n}, \boldsymbol{R}\right) & =i v_{F} \delta\left(x_{1}-x_{2}\right), \\
\hat{g}_{\varepsilon}\left(x_{1}, x_{2} \mid \boldsymbol{n}, \boldsymbol{R}\right)\left(-i v_{F} \frac{\partial}{\partial x_{2}}-\Phi\left(\boldsymbol{r}_{2}\right)+\hat{H}_{\varepsilon, \boldsymbol{n}}\left(\boldsymbol{r}_{2}\right)\right) & =i v_{F} \delta\left(x_{1}-x_{2}\right),
\end{aligned}
$$

where the derivative in Eq. 1.12b operates backwards. The $\delta$-functions in the r.h.s. generates the discontinuity required by Eqs. (1.10).

In Eq. (1.12), $\Phi$ is the gauge invariant scalar potential, and $\hat{H}_{\varepsilon, \boldsymbol{n}}$ is $2 \times 2$ traceless matrix,

$$
\begin{gathered}
\hat{H}_{\varepsilon, \boldsymbol{n}}=\hat{h}_{\varepsilon, \boldsymbol{n}}-\hat{\Sigma}_{\varepsilon, \boldsymbol{n}}, \\
\hat{h}_{\varepsilon, \boldsymbol{n}}=\left(\begin{array}{lr}
\varepsilon-\boldsymbol{v}_{F} \cdot \boldsymbol{p}_{s} & -\Delta_{\boldsymbol{n}} \\
\Delta_{\boldsymbol{n}}^{*} & -\varepsilon+\boldsymbol{v}_{F} \cdot \boldsymbol{p}_{s}
\end{array}\right), \boldsymbol{v}_{F}=v \boldsymbol{n},
\end{gathered}
$$

where $\Delta_{n}$ is the order parameter (which may depend on the direction $\boldsymbol{n}$ ), and $\boldsymbol{p}_{s}=-\frac{e}{c} \boldsymbol{A}, \boldsymbol{A}$ being the vector potential, and $\hat{\Sigma}$ is built of the impurity self-energy as well as the part of the electron-phonon self-energy not included to the self-consistent field $\Delta$. Below, we omit $\boldsymbol{R}, \boldsymbol{n}$ and $\varepsilon$ for brevity.

The boundary condition to Eq. (1.12) on an infinite trajectory from Eq. (1.3),

$$
\hat{g}\left(x_{1}, x_{2}\right) \rightarrow 0 \quad \text { for } \quad\left|x_{1}-x_{2}\right| \rightarrow \infty .
$$

The equations of motion Eq. $\sqrt{12}$ ) can be solved by factorizing the Green's function 12 . For this, one finds linear independent two-component solutions $\phi_{ \pm}(x)=$ $\left(\begin{array}{l}u_{ \pm} \\ v_{ \pm}\end{array}\right)$to the equation

$$
\left(i v_{F} \frac{\partial}{\partial x}+\hat{H}\right) \phi_{ \pm}=0, \quad, \quad \phi_{ \pm}(x) \rightarrow 0 \quad, \quad x \rightarrow \pm \infty
$$

where $H$ is in the operator on the l.h.s. of Eqs. (1.12); This equation is essentially the same as the one first derived by Andreev in the theory of reflection on the NSin terface. The ratio $u_{ \pm} / v_{ \pm}$obeys the Riccati equation 11.25 , which is most convenient for numerics. Known solution to the Riccati equation for $u_{ \pm} / v_{ \pm}$, one readily finds full $\phi_{ \pm}$as explained in 5 .

The solutions to the linear equation Eq. (1.14) can always be normalized as

$$
\bar{\phi}_{-} \phi_{+}=1
$$




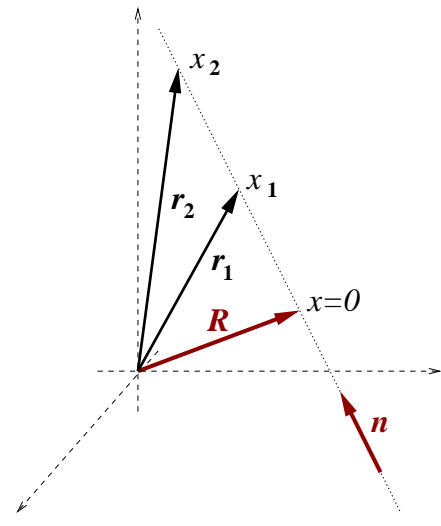

FIG. 4: Classical trajectory. When no interfaces or boundaries are present the trajectory is a straight line. It can be specified by an initial point $\boldsymbol{R}$ and the unit vector $\boldsymbol{n}$, which shows the direction of the trajectory. Introducing the coordinate along the trajectory $x$, any point $r$ on the trajectory can be written as $\boldsymbol{r}=\boldsymbol{R}+x \boldsymbol{n}$.

where here and below the bar on the top of a 2-component column, $\psi$, or a matrix $X$, has the following meaning

$$
\bar{\psi}=\psi^{T} \tau_{y} \frac{1}{i} \quad, \quad \bar{X}=\tau_{y} X^{T} \tau_{y}
$$

where $T$ stands for transposition and $\tau_{y}$ is the Pauli matrix. Taking advantage of the property $H=-\bar{H}$, one can prove that the normalization in Eq. (1.15) is generally compatible with Eq. (1.14).

Denote also

$$
\phi_{\nu}^{\ddagger}=\nu \bar{\phi}_{-\nu} \quad, \quad \nu= \pm .
$$

Then,

$$
\phi_{\nu}^{\ddagger} \phi_{\nu^{\prime}}=\delta_{\nu \nu^{\prime}} \quad, \quad \sum_{\nu} \phi_{\nu} \phi_{\nu}^{\ddagger}=\hat{1}
$$

as immediately follows from the normalization Eq. (1.15) and the definitions in Eq. (1.16).

Now, the solution to Eq. (1.12) reads

$$
\hat{g}\left(x_{1}, x_{2}\right)=\left\{\begin{array}{rrr}
\phi_{+}\left(x_{1}\right) \phi_{+}^{\ddagger}\left(x_{2}\right) & , \quad x_{1}>x_{2} ; \\
-\phi_{-}\left(x_{1}\right) \phi_{-}^{\ddagger}\left(x_{2}\right) & , \quad x_{1}<x_{2} .
\end{array}\right.
$$

In the standard formulation of the quasiclassical technique after Eilenberger, one deals with the 1-point Green's function $\hat{g}(r)$, which is related to the 2-point Green's function a 12

$$
\hat{g}(x)=\hat{g}_{+}(x)+\hat{g}_{-}(x)
$$

where

$$
\hat{g}_{ \pm}(x)=\hat{g}\left(x_{1}=x \pm 0, x_{2}=x \mp 0\right)
$$

Combining Eqs. (1.12a), and (1.12b), one re-derives the well-known Eilenberger equation $\amalg$,

$$
i \boldsymbol{v}_{F} \cdot \nabla \hat{g}+\hat{H} \hat{g}-\hat{g} \hat{H}=0,
$$

for the matrix function $\hat{g}$. The expressions for observables like the charge current and the density of states are presented in Section A.

In the factorized representation Eq. (1.18), the normalization condition for the Eilenberger function Eq. (1.19),

$$
(\hat{g})^{2}=\hat{1}
$$

immediately follows from the orthonormality relations in Eq. (1.17).

To trace the general features needed for the validity of Eq. (1.20), we first note that

$$
\hat{g}_{+}(x)-\hat{g}_{-}(x)=\hat{1}
$$

as is dictated by the $\delta$-function in the r.h.s. of Eq. (1.12). Taking square of this equation and Eq. (1.19), one gets the normalization condition Eq. (1.20) provided

$$
g_{+}(x) g_{-}(x)=g_{-}(x) g_{+}(x)=0 .
$$

The proof of these relations presented in Ref.22, essentially uses the asymptotics in Eq. (1.13), which makes sense only for an infinite trajectory. Besides, the proof tacitly presumes that the quasiclassical equations of motion Eq. (1.12) are applicable everywhere on the trajectory. The latter assumption is not valid if the trajectory under consideration is crossed by another trajectory - the trajectory crossing happens, for instance, on partially transparent interfaces. As shown in Ref.5, the trajectory crossings do not influence the normalization provided the crossings do not change the simple onedimensional topology of the trajectories. It is the purpose of the present paper to analyse more general cases.

After the review of the two-point quasiclassical Green's function method, we will extend the method to the case when the classical trajectories have more complicated topology. In the standard theory, the Fermi wave length scale does not enter the theory after the factorization in Eq. (1.8) has been carried out. As we will see later, this disentanglement of the different scales can not be performed in the case of more complicated geometry.

\section{GREEN'S FUNCTION ON A LOOP}

The theory reviewed in the previous section refers to the situation when the trajectory is a straight line. One can easily generalise the derivation to the case when the particle moves in a smooth quasiclassical potential and its trajectories are not straight lines. If the trajectory corresponds to the infinite motion, no important changes in the theory occur: one can use Eq. (1.12) with the understanding that $x$ is the coordinate along the path. If the orbit is closed, the situation is very different. Physically, the obvious difference is that if the particle moves along a trajectory it eventually comes again to the starting point; then, statements like $x_{1}>x_{2}$ do not have unequivocal 
sense. Also, the boundary condition in Eq. (1.13), without which the equation of motion Eq. (1.12) do not have a unique solution, becomes meaningless.

Of course, in a clean homogeneous metal one can hardly imagine any smooth loop-like trajectory because any potential is screened by mobile charges. Actually, we have in mind to consider loops created as a result of multiple reflections by interfaces. However, for the sake of presentation, it is convenient to us to analyse first a smooth closed trajectory. Besides, the problem is of general interest.

Consider a closed loop trajectory shown in Fig. 5 . We accept it without derivation that Eq. (1.12) may serve as the equation of motion for the two point trajectory Green's function. What is needed is the boundary condition replacing Eq. (1.13).

First, we recall that the full propagator is given by $\mathbb{G}$ in Eq. (1.8),

$$
i v_{F} \mathbb{G}\left(x_{1}, x_{2}\right) \tau_{z}=g\left(x_{1}, x_{2}\right) e^{i p_{F}\left(x_{1}-x_{2}\right)},
$$

omitting the redundant superscripts \pm . Consider the Green's function at nearly coinciding arguments, $\mathbb{G}\left(x_{1}=x_{2}+0, x_{2}\right)$. Using equation of motion, one can find $\mathbb{G}\left(x_{1}, x_{2}\right)$ advancing $x_{1}$ in the direction of arrows in Fig.5. Eventually, one gets again close to $x_{2}$ when $x_{1}$ reaches the value $x_{2}+\mathcal{L}-0, \mathcal{L}$ being the circumference of the loop. On the other hand, the point $x_{1}=x_{2}+\mathcal{L}-0$ is the same physical point as $x_{1}=x_{2}-0$. From the requirement that the Green's function is uniquely defined, one gets the following boundary condition

$$
\mathbb{G}\left(x_{1}=x_{2}+\mathcal{L}, x_{2}\right)=\mathbb{G}\left(x_{1}=x_{2}-0, x_{2}\right) .
$$

From here one gets the following boundary condition for the quasiclassical Green's function

$$
e^{i p_{F} \mathcal{L}} \hat{g}\left(x_{2}+\mathcal{L}, x_{2}\right)=\hat{g}\left(x_{2}-0, x_{2}\right)
$$

where $\hat{g}\left(x_{2}+\mathcal{L}, x_{2}\right)$ is the quasiclassical Green's function continued with the help of Eq. (1.12) from $x_{1}=x_{2}+0$ along the loop of the length $\mathcal{L}$ to the point $x_{1}=x_{2}+\mathcal{L}$.

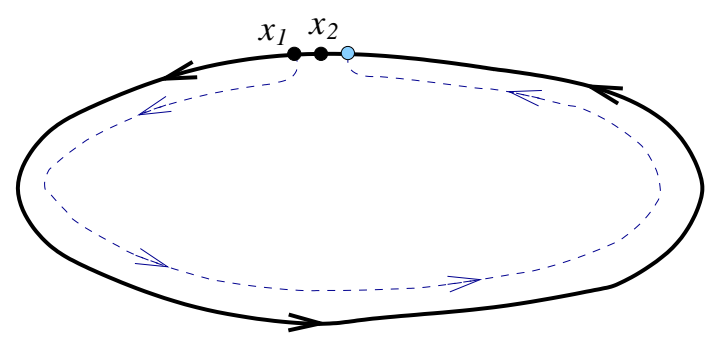

FIG. 5: A loop. The Green's function is a function of the two coordinates along the loop $x_{1}$ and $x_{2}$. As shown in the picture, advancing gradually the first argument from the position $x_{1}=$ $x_{2}+0$ in the direction of its increase, one approaches the point marked by the dashed circle which locally correponds to the coordinate $x_{1}=x_{2}-0$.
As the next step, we derive formally exact expression for the 1-point quasiclassical Green's function via the quasiclassical evolution operator $U\left(x, x^{\prime}\right)$. The latter solves the homogeneous part of Eq. (1.12),

$$
\left(i v_{F} \frac{\partial}{\partial x}+\hat{H}\right) U\left(x, x^{\prime}\right)=0,
$$

with the initial condition $U(x, x)=1$. A formal solution to Eq. (2.3) reads

$$
U\left(x, x^{\prime}\right)=\exp \left[\frac{i}{v_{F}} \int_{x^{\prime}}^{x} d x \hat{H}\right]
$$

where $x$-ordering of the matrices $\hat{H}(x)$ is implied. Properties of $U$ has been discussed in many papers, see e.g.11. In particular, $\operatorname{det} U=1$, and

$$
U\left(x, x^{\prime}\right) \bar{U}\left(x, x^{\prime}\right)=\hat{1}
$$

The expression for the evolution operator in the Riccati equation technique is presented in Section B (see also E) $^{\text {). }}$.

Denote

$$
U_{x}=U(x+\mathcal{L}, x) \quad, \quad \mathcal{U}_{x}=e^{i p_{F} \mathcal{L}} U_{x} .
$$

Then $\hat{g}(x+\mathcal{L}, x)=U_{x} \hat{g}(x+0, x)$ and the boundary condition Eq. (2.2) acquires the form

$$
\mathcal{U}_{x} \hat{g}(x+0, x)=\hat{g}(x-0, x)
$$

This equation together with

$$
\hat{g}(x+0, x)-\hat{g}(x-0, x)=\hat{1}
$$

allows one to obtain the expression for the one-point Green's functions via the evolution operator:

$$
\begin{aligned}
\hat{g}_{+}(x) & =\frac{1}{1-\mathcal{U}_{x}} \\
\hat{g}_{-}(x) & =\frac{\mathcal{U}_{x}}{1-\mathcal{U}_{x}} \\
\hat{g}(x) & =\frac{1+\mathcal{U}_{x}}{1-\mathcal{U}_{x}}
\end{aligned}
$$

For illustration, we consider some simple applications of this result.

\section{Homogeneous loop}

Suppose the "Hamiltonian" $\hat{H}$ in Eq. (2.3) does not depend on the coordinate. The evolution operator $U_{x}$ can be easily found from Eq. (2.4). For this, we present $\hat{H}$ as

$$
\hat{H}=\xi \psi_{+} \psi_{+}^{\ddagger}-\xi \psi_{-} \psi_{-}^{\ddagger}
$$

where $\psi_{ \pm}$are the eigenfunction of $\hat{H}$,

$$
\hat{H} \psi_{ \pm}= \pm \xi \psi_{ \pm} \quad, \quad \Im \xi>0
$$


normalized in accordance with Eq. (1.17). From Eq. (2.4), the loop evolution operator reads

$$
U_{x}=u \psi_{+} \psi_{+}^{\ddagger}+\frac{1}{u} \psi_{-} \psi_{-}^{\ddagger},
$$

independent on $x$. Parameter $u$,

$$
u=e^{\frac{i \xi \mathcal{L}}{v_{F}}}
$$

shows the variation of the phase of the wave and its absolute value upon propagation around the loop. The modulus of $u,|u|=\exp \left[-\mathcal{L} \Im \xi / v_{F}\right]<1$, is less than unity.

The one-point Green's function Eq. (2.6b) reads

$$
\hat{g}=\frac{1+u e^{i p_{F} \mathcal{L}}}{1-u e^{i p_{F} \mathcal{L}}} \psi_{+} \psi_{+}^{\ddagger}-\frac{1+u e^{-i p_{F} \mathcal{L}}}{1-u e^{-i p_{F} \mathcal{L}}} \psi_{-} \psi_{-}^{\ddagger} .
$$

This result is rather transparent physically. The Green's function has a contribution from (quasi)electrons and (quasi) holes given by the first and second terms, respectively. The (retarded) Green's function is built of waves decaying in the direction of propagation, and one can use attenuation of the wave, $|u|$, as a meter which shows the length of the path the particle has travelled. Expanding the Green's function in Eq. (2.10) with respect to $u$,

$$
\begin{aligned}
\hat{g} & =\psi_{+} \psi_{+}^{\ddagger}\left(1+u e^{i p_{F} \mathcal{L}}+\left(u e^{i p_{F} \mathcal{L}}\right)^{2}+\ldots\right) \\
& -\psi_{-} \psi_{-}^{\ddagger}\left(1+u e^{-i p_{F} \mathcal{L}}+\left(u e^{-i p_{F} \mathcal{L}}\right)^{2}+\ldots\right) .
\end{aligned}
$$

one gets information on the paths contributing the propagator and their length. Looking at the first line, one sees that the Green's function has a contribution which corresponds to the electron-like quasiparticle $\psi_{+}$circling the loop in the positive direction (where the phase in Fermi momentum factor increases); the paths have the winding numbers $0,1,2 \ldots$ and the lengths $0, \mathcal{L}, 2 \mathcal{L}, \ldots$, respectively. The second line shows the contribution due to holes: Decaying in the direction of propagation, they circle the loop in the negative directipm as can be read off the Fermi momentum phase factor 16 . In the limit of an infinite loop, when $u \rightarrow 0$, one restores the well-know result for a homogeneous superconductor.

The electron and hole parts in Eq. (2.10) have poles at the energies where the denominators have zeroth. In the ballistic case, when the non-Hermitian part of $H$ is only due to the infinitesimal $\delta$ in $\varepsilon+i \delta$, the poles give the bound states which correspond to the usual BohrSommerfeld quantisation for closed orbits. Impurities, generating the imaginary part of the self-energy, reduce $u$ and broaden the bound states. For quantities averaged with respect to disorder, the closed topology of the orbit is seen only if the orbit is shorter than the mean free path. At Matsubara frequencies $\varepsilon=i \omega_{n}$, the orbit must be shorter than the coherence length $v_{F} / \omega_{n}$.

A qualitative difference between the cases of a loop and an infinite trajectory, is clearly seen in the expansion of
$\hat{g}_{+}$Eq. (2.6a),

$$
\begin{aligned}
\hat{g}_{+} & =\psi_{+} \psi_{+}^{\ddagger}\left(1+u e^{i p_{F} \mathcal{L}}+\left(u e^{i p_{F} \mathcal{L}}\right)^{2}+\ldots\right) \\
& -\psi_{-} \psi_{-}^{\ddagger}\left(u e^{-i p_{F} \mathcal{L}}+\left(u e^{-i p_{F} \mathcal{L}}\right)^{2}+\ldots\right)
\end{aligned}
$$

One sees that, unlike the case of an infinite trajectory, the hole contributes to $\hat{g}_{+}$. For this, the hole must circle the loop at least once. The reason for the difference between electron and hole is physically clear. Indeed, by definition, $g_{+}(x)$ is the propagator $g(x+0, x)$ from the source at the point $x$ to the neighbouring point $x+0$. The source (i.e. the r.h.s of Eqs. 1.12a) creates electron which propagates in the positive direction, and the hole moving in the negative direction, nominally, away from the point $x+0$. However, having made a full turn(s) around the loop, the hole reaches the point $x+0$, and, therefore, does contribute to $\hat{g}_{+}$. Ultimately, these "wrong" processes where the excitation effectively moves in the wrong direction, lead to the violation of the normalization condition for the quasiclassical Green's function.

\section{Normalization condition}

To check the normalization condition, we note that $\hat{g}^{2}-1$ can be conveniently presented as

$$
\hat{g}^{2}-1=4 \hat{g}_{+} \hat{g}_{-}
$$

From Eq. (2.6), $\hat{g}_{+} \hat{g}_{-}=\mathcal{U}_{x} /\left(1-\mathcal{U}_{x}\right)^{2}$, or in the eigenfunction representation,

$$
\hat{g}_{+} \hat{g}_{-}=\frac{u e^{i p_{F} \mathcal{L}}}{\left(1-u e^{i p_{F} \mathcal{L}}\right)^{2}} \psi_{+} \psi_{+}^{\ddagger}+\frac{u e^{-i p_{F} \mathcal{L}}}{\left(1-u e^{-i p_{F} \mathcal{L}}\right)^{2}} \psi_{-} \psi_{-}^{\ddagger} .
$$

One can see that the orthogonality and normalization condition do not hold for the Green's function on a loop. Of course, this is not informative from the point of view of checking the validity of the quasiclassical theory which deals with coarse-grained objects. Note, however, the r.h.s. of Eq. (2.12) vanishes when $u \rightarrow 0$, restoring the normalization Eq. (1.20) in the limit of an infinite loop.

\section{Coarse-grained average}

Observables like the local density of states (DOS) calculated with the help of the Green's function in Eq. (2.6b) or Eq. (2.10), may show wild fluctuations as a function of position or energy. Indeed, in a ballistic system the DOS is given by a series of closely spaced $\delta$-function peaks due to the space quantization in the loop. On a low-spatial resolution, when loops with slightly different $\mathcal{L}$ contribute to observables, DOS becomes an essentially smooth function. The summation over the loops in a small interval of $\delta \mathcal{L}$ is equivalent to averaging with respect to the phase of $\varphi=p_{F} \mathcal{L}$ provided $p_{F} \delta \mathcal{L}>1$ (c®1). 
In the representation Eq. (2.10) the integration is easily performed and the coarse-grained Green's function $\hat{g}_{\text {coarse }}$ is simply

$$
\hat{g}_{\text {coarse }}=\psi_{+} \psi_{+}^{\ddagger}-\psi_{-} \psi_{-}^{\ddagger} .
$$

For a homogeniuos loop, this expression coincides with that for an infinite trajectory.

This result is readily generalised to the inhomogeneous loop. Indeed, the evolution matrix can be always presented as in Eq. (2.8) because it is the only form compatible with Eq. (2.5). Unlike the homogeneous case, $\psi_{ \pm}$ are $x$-dependent and there is no simple way to find them. Operationally, $\psi_{ \pm}$are eigenfunctions of $U_{x}$ and $u$ and $1 / u$ its eigenvalues (the latter are global $x$-independent values). Without lost of generality, $|u|<1$ and $\psi_{+}$is the eigenfunction corresponding to the smallest eigenvalue of $U_{x}$. Then, again one comes to Eq. (2.13). Of course, the effective wave functions $\psi_{ \pm}$depend on the coordinate as well as the length the loop $\mathcal{L}$. The normalization condition is granted by the orthonormality relation in Eq. (1.17).

We see that the coarse-grained averaging eliminates "wrong" paths in Eq. (2.11) and the averaged Green's function can be found from the standard quasiclassical theory. However, as we see later, alternative paths may survive the averaging in more complicated geometries.

\section{DOUBLE LOOP}

In this section, we generalise the theory to the case of two loops tied by a knot, see Fig. 6 .

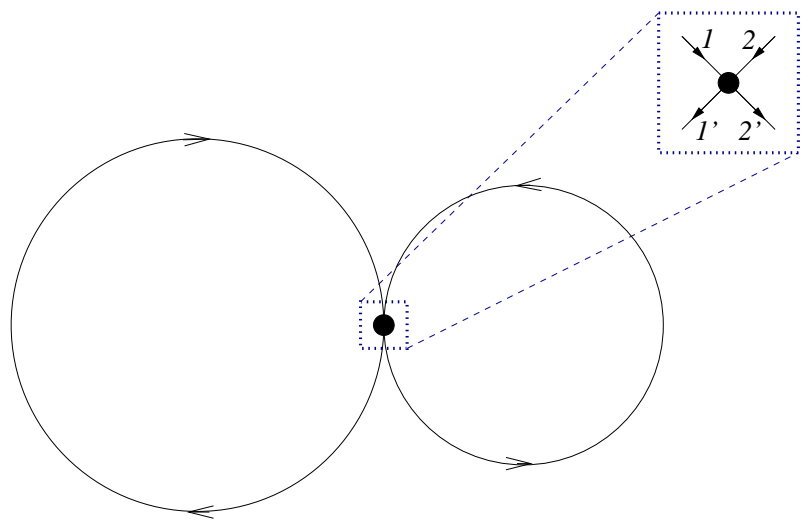

FIG. 6: Two coupled loops. The arrows show the direction in which the Fermi phase increases (the momentum direction). The filled circle stands for the knot which ties the loops allowing for the coupling of the loops. The amplitudes of the waves at the points adjacent to the knot, 1, 1' on the left loop and 2, 2' on the right loop, are related by the $S$-matrix in accordance with Eq. (3.2).

As before, we denote the propagator $\hat{g}\left(x_{1}, x_{2}\right)$ with the understanding that $x_{1}$ and $x_{2}$ may stand for either $x^{(l)}$ or $x^{(r)}$, the coordinates of the points on the left and right loops, respectively. The initial and final points of the propagator may be on the same loop or belong to the different loops, see Fig. 6. The coordinates $x^{(l, r)}$ are counted from the knot in the direction of the corresponding arrow. They assume values $0<x^{(l, r)}<\mathcal{L}^{(l, r)}, \mathcal{L}^{(l, r)}$ being the circumference of the left or right loop. The equations of motion within each of the loops are given by Eq. (1.12). We couple the loops with a phenomenological unitary $S$-matrix,

$$
S=\left(\begin{array}{cc}
\rho & t \\
t & \rho
\end{array}\right)
$$

where parameter $t$ describes the inter-loop coupling ("transmission amplitude") and $\rho$ is the intra-loop scattering amplitude. These parameters are taken same for the electron and hole components of the wave function. Apart from the the unitarity condition,

$$
|\rho|^{2}+|t|^{2}=1 \quad, \quad \rho t^{*}+\rho^{*} t=0,
$$

$\rho$ and $t$ are free parameters. In the limit $t=0$, one has two independent loops, and, if $r=0$, the two loops are equivalent to a single loop (with the shape like "8").

Denote $\psi_{1,1^{\prime}, 2,2^{\prime}}$ the (two-components) wave functions at the corresponding point $1,1^{\prime}, 2$ or 2 ', adjacent to the knot coupling the loops (see Fig G). Then, the $S$-matrix expresses the amplitudes of the outgoing waves, $\psi_{1^{\prime}, 2^{\prime}}$,

$$
\left(\begin{array}{l}
\psi_{1^{\prime}} \\
\psi_{2^{\prime}}
\end{array}\right)=\left(\begin{array}{ll}
\rho & t \\
t & \rho
\end{array}\right)\left(\begin{array}{l}
\psi_{1} \\
\psi_{2}
\end{array}\right)
$$

via the incoming wave amplitudes $\psi_{1,2}$.

The formal solution in Eq. (2.6) for the one-point Green's functions $\hat{g}_{ \pm}\left(x^{(l, r)}\right)$ remains valid if the loop evolution operator $\mathcal{U}_{x^{(l, r)}}$ properly accounts for the knot.

Consider for definiteness the right loop. The full loop evolution matrix can be written as the product,

$$
\mathcal{U}_{x}(r)=e^{i p_{F} \mathcal{L}^{(r)}} U^{(r)}\left(x^{(r)}, 0\right) \mathcal{R}^{(r)} U^{(r)}\left(\mathcal{L}^{(r)}, x^{(r)}\right),
$$

where $U^{(r)}\left(x^{(r)}, x^{(r) \prime}\right)$ is the in-loop evolution matrix found from Eq. (2.3), and $\mathcal{R}^{(r)}$ (the transfer matrix) accounts for the propagation through the knot from the point 2 to the point 2' (see Fig. 6). The latter can be evaluated with the help of Eq. (3.2) by the following arguments.

While calculating the one-point Green's function for the right loop, the waves in the left loop obey homogeniuos equation of motion. Then, the amplitudes $\psi_{1}$ (see Fig.(6) and $\psi_{1^{\prime}}$ are related as

$$
\psi_{1}=\mathcal{U}^{(l)} \psi_{1^{\prime}}
$$

where

$$
\mathcal{U}^{(l)}=e^{i p_{F} \mathcal{L}^{(l)}} U^{(l)}
$$


and $U^{(l)}$ is the quasiclassical evolution matrix in the left loop which advances $x^{(l)}$ from $x^{(l)}=0$ to $x^{(l)}=\mathcal{L}^{(l)}$ :

$$
U^{(l)}=U^{(l)}\left(\mathcal{L}^{(l)}, 0\right) .
$$

Combining Eqs. (3.2), and (3.4), one excludes amplitudes $\psi_{1}$ and $\psi_{1^{\prime}}$, and finds that

$$
\psi_{2^{\prime}}=\mathcal{R}^{(r)} \psi_{2}
$$

where

$$
\mathcal{R}^{(r)}=\frac{1-\frac{\mathcal{U}^{(l)}}{\rho^{*}}}{\frac{1}{\rho}-\mathcal{U}^{(l)}} .
$$

Eq. (1.12) together with Eqs. (3.3), and (3.6), allows one to find the Green's function at any point of the loop.

For instance, at point $2^{\prime}$ and 2 where $x^{(r)}=0$ and $x^{(r)}=\mathcal{L}^{(r)}$, respectively,

$$
\begin{aligned}
\mathcal{U}_{2^{\prime}} & =\left(1-\frac{T}{R} \frac{\rho \mathcal{U}^{(l)}}{1-\rho \mathcal{U}^{(l)}}\right) \rho \mathcal{U}^{(r)} \\
\mathcal{U}_{2} & =\rho \mathcal{U}^{(r)}\left(1-\frac{T}{R} \frac{\rho \mathcal{U}^{(l)}}{1-\rho \mathcal{U}^{(l)}}\right)
\end{aligned}
$$

where $T=1-R$.

Although not obvious from the beginning, the structure of the equations allows one to absorb the phase of $\rho$ to the phase factors $e^{i p_{F} \mathcal{L}^{(l, r)}}$, so that $\rho$ becomes effectively a real positive parameter

$$
\rho \rightarrow \sqrt{R} \quad, \quad R=|\rho|^{2} .
$$

Then Eqs. (3.7), and (3.8) can be presented as

$$
\begin{aligned}
\mathcal{U}_{2} & =\sqrt{R} \mathcal{U}^{(r)}-T \mathcal{U}^{(r)} \frac{\mathcal{U}^{(l)}}{1-\sqrt{R} \mathcal{U}^{(l)}}, \\
\mathcal{U}_{2^{\prime}} & =\sqrt{R} \mathcal{U}^{(r)}-T \frac{\mathcal{U}^{(l)}}{1-\sqrt{R} \mathcal{U}^{(l)}} \mathcal{U}^{(r)} .
\end{aligned}
$$

Inserting these expressions for the full evolution matrices into Eq. (2.6a), one gets after some transformation the Green's function $\hat{g}_{+}$at the points 2 and $2^{\prime}$ :

$$
\begin{aligned}
\hat{g}_{+2} & =\left(1+T \frac{\mathcal{U}^{(r)}}{1-\sqrt{R} \mathcal{U}^{(r)}} \frac{\mathcal{U}^{(l)}}{1-\sqrt{R} \mathcal{U}^{(l)}}\right)^{-1} \frac{1}{1-\sqrt{R} \mathcal{U}^{(r)}} \\
\hat{g}_{+2^{\prime}} & =\frac{1}{1-\sqrt{R} \mathcal{U}^{(r)}}\left(1+T \frac{\mathcal{U}^{(l)}}{1-\sqrt{R} \mathcal{U}^{(l)}} \frac{\mathcal{U}^{(r)}}{1-\sqrt{R} \mathcal{U}^{(r)}}\right)^{-1}
\end{aligned}
$$

These expressions for the Green's function are exact within the Andreev approximation where the normal metal spectrum is linearised in the vicinity of the Fermi surface; by a different method, similar formulae for a sandwich have been previously derived in 12 . In particular, they allow for the space quantisation due to the normal reflection (in addition to the Andreen levels grasped by the conventional quasiclassical theory 4 ). The evolution matrices $\mathcal{U}^{(r, l)}$ defined by Eq. (3.5), can be evaluated in the standard quasiclassical scheme, for instance, in the Riccati equation technique (see Sect B).

\section{A. Checking the normalization condition}

Now, we want to demonstrate that unlike the simple loop case, the normalization condition for the Green's function for a double loop is violated even on the coarsegrained level.

As before, the coarse grain averaging amounts to the integration of the Green's functions with respect to the phases factors

$$
e^{i \varphi^{(l, r)}}=e^{i p_{F} \mathcal{L}^{(l, r)}}
$$

$\mathcal{L}^{(l, r)}$ being the length of the corresponding loop. In principle, the integration can be performed in terms of the elliptic integrals as in 11 . Instead, we use an approximate procedure which allows one to get better control of the physics behind the violation of normalization.

Analogously to Eq. (2.8), we present the evolution matrices entering Eq. (3.9) as

$$
\mathcal{U}^{(l, r)}=e^{i p_{F} \mathcal{L}^{(l, r)}}\left(u \psi_{+} \psi_{+}^{\ddagger}+\frac{1}{u} \psi_{-} \psi_{-}^{\ddagger}\right)^{(l, r)}
$$

where $u^{(l, r)}$ are given by Eq. (2.9) for each of the loops. Algebra is greatly simplified if one uses the matrix representation where the evolution in the right (or left) loop is diagonal, i.e.

$$
\mathcal{U}^{(r)}=e^{i p_{F} \mathcal{L}^{(r)}}\left(\begin{array}{ll}
u^{(r)} & 0 \\
0 & \frac{1}{u^{(r)}}
\end{array}\right) .
$$


This can be always achieved by the transformation of all the matrices,

$$
\hat{X} \rightarrow \mathcal{O} \hat{X} \mathcal{O}^{-1}
$$

with the matrix $\mathcal{O}$, which diagonalizes $\mathcal{U}^{(r)}$. In this representation, the right loop is effectively a normal metal.

Up to a normalisation factor, the eigenfunction $\psi_{ \pm}^{(l)}$ of $\mathcal{U}^{(l)}$ can be written as:

$$
\psi_{+}^{(l)}=\left(\begin{array}{c}
1 \\
A_{+}
\end{array}\right) \quad, \quad \psi_{-}^{(l)}=\left(\begin{array}{c}
A_{-} \\
1
\end{array}\right) .
$$

In this representation, $A_{ \pm}$has a simple physical meaning: $A_{+}\left(A_{-}\right)$are amplitudes of the Andreev reflection of the electron (hole) excitation for the ideal case $t=1, \rho=0$ (a totally transparent interface).

The left layer evolution matrix reads

$$
\mathcal{U}^{(l)}=e^{i p_{F} \mathcal{L}^{(l)}}\left(\frac{1}{2}\left(1+g_{0}^{(l)}\right) u^{(l)}+\frac{1}{2}\left(1-g_{0}^{(l)}\right) \frac{1}{u^{(l)}}\right)
$$

where

$$
g_{0}^{(l)}=\frac{1}{1-A_{+} A_{-}}\left(\begin{array}{lr}
1+A_{+} A_{-} & -2 A_{-} \\
2 A_{+} & -1-A_{+} A_{-}
\end{array}\right) .
$$

We plug these relations into Eq. (3.9) and perform an expansion in series of $u^{(l, r)}$. From Eqs. (3.12), and (3.15), one sees that each power of $u^{(l, r)}$ brings with it $e^{+i \varphi^{(l, r)}}$ (electron) or $e^{-i \varphi^{(l, r)}}$ (hole). Accordingly, the expansion for a Green's function $G$ has the following structure

$$
G=\sum_{k, n=0}^{\infty}\left(u^{(l)}\right)^{k}\left(u^{(r)}\right)^{n} \sum_{p=-k}^{k} \sum_{q=-n}^{n} e^{i p \varphi^{(l)}} e^{i q \varphi^{(r)}} G_{k, n}^{p, q}
$$

Each term which can be associated with the corresponding process where the particle starts moving from an initial point and returns back having moved around the loops. En route, it undergoes electron-hole conversion as a result of the Andreev reflection on the knot. The Fermi momentum phase increases when the particle moves in the direction of the arrows in Fig. 6 (as the electron) and decreases otherwise (for the hole). The total acquired phase is controlled by the history of the electronhole conversion. The meaning of the integers $k, n, p$, and $q$ in in Eq. (3.16) is the following. For instance, $k$ shows the total length of the path $k \mathcal{L}^{(l)}$ in the left loop. If the particle have circled the loop $k_{e}$ times as electron and $k_{h}$ times as hole $\left(k=k_{e}+k_{h}\right)$, the acquired Fermi momentum phase corresponds to $p$ in Eq. (3.16) equal to $p=k_{e}-k_{h}$. Parameters $n$ and $q$ have analogous meaning for the right loop.

On the coarse-grain level of description, one performs averaging within an interval of the loop length $\delta \mathcal{L} \ll \mathcal{L}$ which is wide enough to average the phase factors in
Eq. (3.11) to zero. Independent averaging with respect to length of the left and right loops, amounts to ignoring any term in Eq. (3.16) other than $p=q=0$. For certain geometries, the phase factors $e^{i \varphi^{(l)}}$ and $e^{i \varphi^{(r)}}$ cannot be considered independent. This occur if $\mathcal{L}^{(l)}=\mathcal{L}^{(r)}$, or more generally, the lengths of the loops are commensurable, $N \mathcal{L}^{(l)}=M \mathcal{L}^{(r)}, N, M=1,2 \ldots$ In this case, the terms $p= \pm N, q=\mp M$ (and their multiples) also contribute to the coarse grain average.

For definiteness, we consider the Green's function, $\hat{g}_{2}$, corresponding to the point 2 in the immediate vicinity of the knot (see Fig. 6). The loops are assumed to be incommensurable. The result of the calculation of the averaged Green's function $\left\langle\hat{g}_{2}\right\rangle$ can be presented as follows:

$$
\left\langle\hat{g}_{2}\right\rangle=\hat{G}_{q c}+\hat{G}_{n q c}
$$

where $\hat{G}_{q c}$ is of the quasiclassical Maki-Schopohl form,

$$
\hat{G}_{q c}=\frac{1}{1-\alpha \beta}\left(\begin{array}{lr}
1+\alpha \beta & -2 \alpha \\
2 \beta & -(1-\alpha \beta)
\end{array}\right)
$$

with

$$
\begin{aligned}
\alpha= & \left.A_{-} \gamma T u^{(r)^{2}}\left(1-\gamma^{2} \mu \nu T u^{(l)}\right)^{2}\right)+\ldots \\
\beta= & A_{+} \gamma T\left(\left(1-\gamma^{2} \mu \nu T u^{(l)^{2}}\right)\left(1+\gamma^{2} \mu^{2} R u^{(r)^{2}}\right)\right. \\
& \left.+T^{2} \gamma^{4} u^{(l)^{2}} u^{(r)^{2}} \mu^{2}\left(\nu^{2}+4 R A_{-} A_{+}\right)\right)+.(3.18
\end{aligned}
$$

where

$$
\mu=1-A_{+} A_{-}, \nu=1+R A_{+} A_{-}, \gamma=\frac{1}{1-R A_{+} A_{-}}
$$

these combinations of the Andreev amplitudes are wellknown in the theory of an imperfect NS-interface 17 . The second term in the r.h.s. of Eq. (3.17), $\hat{G}_{n q c}$, reads

$$
\begin{aligned}
\hat{G}_{n q c} & =4 \gamma^{4} \mu^{2} R T^{2} u^{(r) 2} u^{(l) 2} \\
& \times\left(\begin{array}{cc}
A_{+} A_{-} & 0 \\
A_{+} \gamma\left(2 T A_{+} A_{-}-\mu \nu\right) & -A_{+} A_{-}
\end{array}\right)+\ldots
\end{aligned}
$$

Squaring the coarse-grained Green's function, one gets

$$
\left\langle g_{2}\right\rangle^{2}-\hat{1}=8 A_{+} A_{-} \mu^{2} \gamma^{4} R T^{2} u^{(l)^{2}} u^{(r)^{2}} \hat{1}+\ldots
$$

These expressions are valid up to $u^{(r) 2}$ and $u^{(l) 2}$, their product included.

One sees that the normalization condition is not restored by the coarse-grain averaging as in the case of a single loop. From the structure of the r.h.s. of Eq. (3.19), one may infer that the process responsible for the violation of the normalization condition is the one where the particle follows an $\infty$-like path twice along the loops, each in the opposite direction; such a path is $\left(u^{(l)} u^{(r)}\right)^{2}$ long and the change of the direction of motion requires two 
sequential Andreev reflections, $A_{+}$and $A_{-}$. In the limits $R \rightarrow 0$ or $T \rightarrow 0$, one restores the single loop situation and the normalization condition. Note also, the normalization is not violated if $A_{ \pm}=0$, i.e. the electron-hole conversion is absent (in the normal metal case or if the loops are made of the same material).

\section{SANDWICH}

Now we are in position to apply the theory of previous sections to a physical system. We consider a two-layer system shown in Fig.1 formed by two superconducting layer, left and right. The properties of the layers are specified by their thicknesses $a^{(l, r)}$ and the complex order parameter $\Delta^{(l, r)}$ (they are considered as input, and the self-consistency condition is not discussed). We assume that the order parameter depends only on the coordinate $x$ perpendicular to the layer plane.

The Gor'kov equation for the matrix Green's function $\mathbb{G}_{\varepsilon}\left(\boldsymbol{r}_{1}, \boldsymbol{r}_{2}\right)$ reads

$$
(\varepsilon-\mathbb{H}-\mathbb{V}) \mathbb{G}_{\varepsilon}=\mathbb{1}
$$

where $\mathbb{H}$ is defined in Eq. (1.2) and $\mathbb{V}$ is the potential describing the the interface. As before, we assume the parabolic spectrum, i.e.

$$
\hat{\xi}=\frac{\hat{\boldsymbol{p}}^{2}}{2 m}-\frac{p_{F}^{2}}{2 m} .
$$

Due to the in-plane translational symmetry, the Green's function can be presented as

$$
\mathbb{G}_{\varepsilon}\left(\boldsymbol{r}, \boldsymbol{r}^{\prime}\right)=\sum_{\boldsymbol{p}_{\| \mid}} \mathbb{G}_{\varepsilon}\left(x, x^{\prime} ; \boldsymbol{p}_{\|}\right) e^{i \boldsymbol{p}_{\| \cdot}\left(\boldsymbol{r}-\boldsymbol{r}^{\prime}\right)_{\|}}
$$

To simplify notations, we use below $\mathbb{G}\left(x, x^{\prime}\right)$ as a shorthand for $\mathbb{G}_{\varepsilon}\left(x, x^{\prime} ; \boldsymbol{p}_{\|}\right)$.

The matrix $2 \times 2$ Green's function $\mathbb{G}\left(x, x^{\prime}\right)$ is found from

$$
\left(\varepsilon-\mathbb{H}_{x}\right) \mathbb{G}\left(x, x^{\prime}\right)=\hat{1} \delta\left(x-x^{\prime}\right)
$$

where

$$
\mathbb{H}_{x}=\left(\begin{array}{cc}
\varepsilon-\xi_{x} & -\Delta \\
-\Delta^{*} & \varepsilon+\xi_{x}
\end{array}\right)+\mathbb{V}(x)
$$

and

$$
\xi_{x}=\frac{1}{2 m}\left(\frac{1}{i} \frac{d}{d x}\right)^{2}-\frac{p_{F x}^{2}}{2 m} \quad, \quad \frac{p_{F x}^{2}}{2 m}=\frac{p_{F}^{2}}{2 m} \cos ^{2} \theta
$$

where

$$
\cos ^{2} \theta=\left(1-\frac{p_{\|}^{2}}{p_{F}^{2}}\right)
$$

The boundaries at $x=-a^{(l)}, a^{(r)}$ are assumed impenetrable so that

$\mathbb{G}\left(x_{1}, x_{2}=-a^{(l)}\right.$ or $\left.a^{(r)}\right)=\mathbb{G}\left(x_{1}=-a^{(l)}\right.$ or $\left.a^{(r)}, x_{2}\right)=0$

The Green's function $\hat{G}\left(x, x^{\prime}\right)$ is continuous at $x=x^{\prime}$

$$
\mathbb{G}(x+0, x)=\mathbb{G}(x-0, x),
$$

whereas its derivatives suffer a jump generated by the delta-function in the r.h.s. of Eq.(4.1):

$$
\frac{i \hbar}{2 m}\left[p \hat{G} \hat{\tau}_{z}\right]_{x=x^{\prime}-0}^{x=x^{\prime+0}}=1
$$

If one chooses a particular model for the interface between the layers, e.g. a $\delta$-function barrier, one can solve the Gor'kov equation for the retarded Green's function exactly. The analysis of the full solution shows that the Andreev approximation, greatly simplifying the algebra, faithfully preserve all the physics we are going to discuss. Therefore, we restrict ourself to the Andreev approximation as in Sect. If and III.

As in Section II, we split the Green's function into two pieces Eq. (1.6), $\mathbb{G}^{+}$and $\mathbb{G}^{-}$, which describe propagation of the particle injected in the system with the momentum $+p_{F x}$ and $-p_{F x}$, respectively. Consider for for definiteness the Green's function $\mathbb{G}^{+}$in the right layer. Since the initial direction of the momentum may be changed by the reflection on the outer walls and the interface, instead of Eq. (1.8) one has

$$
\begin{aligned}
i v_{F} \mathbb{G}^{+}\left(x_{1}, x_{2}\right) \tau_{z} & =g^{++}\left(x_{1}, x_{2}\right) e^{i p_{F x}\left(x_{1}-x_{2}\right)} \\
& -g^{+-}\left(x_{1}, x_{2}\right) e^{-i p_{F x}\left(\left(x_{1}+x_{2}-2 a^{(r)}\right.\right.}
\end{aligned}
$$

the function $g^{++}$suffers the jump Eq. (1.10) at the source point $x_{1}=x_{2}$ whereas the secondary wave $g^{+-}$is continuous.

From the boundary condition Eq. (4.2),

$$
g^{++}\left(x_{1}=a^{(r)}, x_{2}\right)=g^{+-}\left(x_{1}=a^{(r)}, x_{2}\right) .
$$

Because of the reflections, the particle moves along the trajectories shown in Fig. 7 .

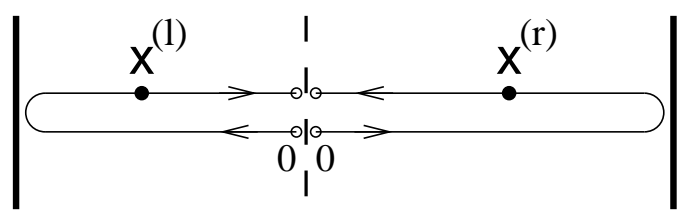

FIG. 7: The trajectory coordinate. In each of the layers the trajectory coordinate $\mathrm{x}^{(l)}$ and $\mathrm{x}^{(r)}$ are counted from the interface along the path of a particle which moves from the interface and is reflected on the surface boundary.

It is convenient to introduce the trajectory coordinates $\mathrm{x}^{(l, r)}$, which assume values in the region $0<\mathrm{x}^{(l, r)}<$ 
$2 a^{(l, r)}$, as

$$
\begin{aligned}
& \mathrm{x}^{r}=\left\{\begin{array}{rr}
x, & 0<\mathrm{x}^{r}<a^{(r)} \\
2 a^{(r)}-x, & a^{(r)}<\mathrm{x}<2 a^{(r)}
\end{array}\right. \\
& \mathrm{x}^{l}=\left\{\begin{array}{rr}
-x, & 0<\mathrm{x}^{l}<a^{(l)} \\
2 a^{(l)}+x, & a^{(l)}<\mathrm{x}<2 a^{(l)}
\end{array}\right.
\end{aligned}
$$

Since the trajectory coordinate space is twice as large as the $x$-space, the two functions of $x$ in Eq. (4.3) may be packed into a single function of the trajectory coordinates $\mathrm{x}_{1}$ and $\mathrm{x}_{2}$, that is

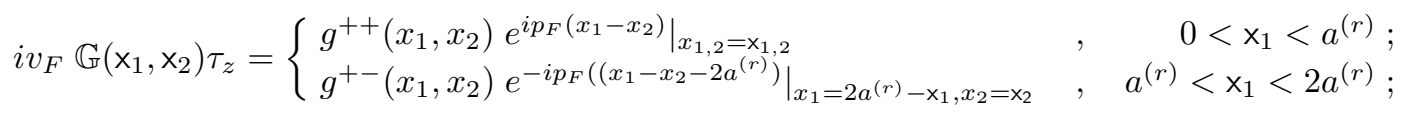

for $\mathrm{x}_{2}<a^{(r)}$. Similar relations, express $\mathbb{G}^{-}\left(x_{1}, x_{2}\right)$ via $\mathbb{G}\left(\mathrm{x}_{1}, \mathrm{x}_{2}\right)$ for the value of $\mathrm{x}_{2}$ in the region $a^{(r)}<\mathrm{x}_{2}<$ $2 a^{(r)}$. The trajectory in the left loop is introduced in the same manner.

Finally, one comes to the representation identical to that introduced for the loop Green's function by Eq. (2.1), i.e.

$$
i v_{F} \mathbb{G}\left(\mathrm{x}_{1}, \mathrm{x}_{2}\right) \tau_{z}=g\left(\mathrm{x}_{1}, \mathrm{x}_{2}\right) e^{i p_{F x}\left(\mathrm{x}_{1}-\mathrm{x}_{2}\right)} .
$$

By virtue of Eq. (4.4), the quasiclassical envelope $g\left(\mathrm{x}_{1}, \mathrm{x}_{2}\right)$ is continuous at the point of the loop corresponding to the outer walls. The envelope satisfies the quasiclassical equations Eq. (1.12) with the understanding $x_{1,2}$ is the trajectory coordinate $\mathrm{x}_{1,2}$.

The coupling of the left and right layers can be described phenomenologically with the help of the S-matrix Eq. (3.1). The simple structure of the S-matrix in Eq. (3.1) corresponds to the assumption the interface is mirror symmetric 18 . In this context, $\rho$ is the amplitude of the reflection by the interface and $t$ is the transmission amplitude. Then, the theory of the loop Green's function presented in Sections II and III can be directly applied to the case of a layer or a sandwich. Within then Andreev approximation, this gives the solution which is "exact" in the sense that it allows for the space quantization (in 11 , this Green's function is called quasiclassical-like).

Schematically, the loops are shown in Fig. 8: Apart from the Fermi momenta phase factor, the propagation of the waves moving towards the interface is described by the evolution operator $U_{1}^{(l)}$ and $U_{2}^{(r)}$ in the left and right layer, respectively. The evolution of the outgoing waves is controlled by $U_{1^{\prime}}^{(l)}$ and $U_{2^{\prime}}^{(r)}$. The in- and out-waves are mixed by the S-matrix, an electron-hole scalar in the Andreev approximation.

The Green's function can be then found from Eq. (3.9) and 3.10 where

$$
\begin{aligned}
\mathcal{U}^{(l, r)} & =e^{\left(2 p_{F x} a^{(l, r)} i+\pi i\right)} U^{(l, r)} \\
U^{(l)}=U_{1}^{(l)} U_{1^{\prime}}^{(l)} & , U^{(r)}=U_{2}^{(r)} U_{2^{\prime}}^{(r)} .
\end{aligned}
$$

The evolution matrix depends on the direction of propagation in an anisotropic superconductor and for this reason $U_{2}^{(r)}$ and $U_{2^{\prime}}^{(r)}$ may differ from each other. The additional $\pi$-phase shift is due to the reflection on the wall

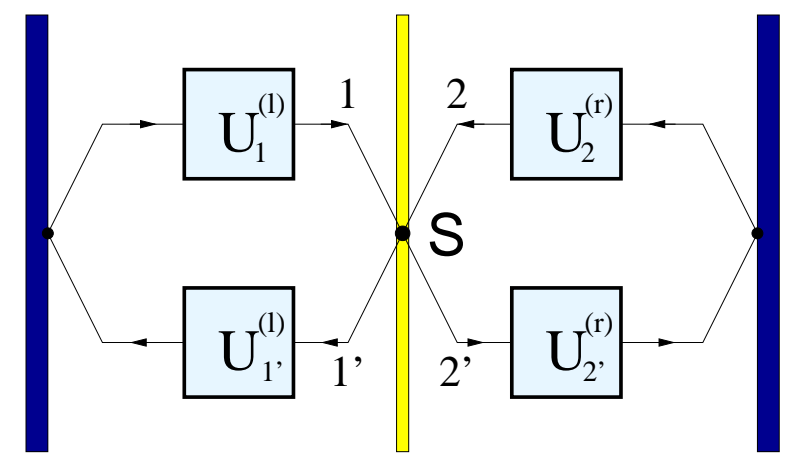

FIG. 8: Waves with definite in-plane momentum in a sandwich. The wave is totally reflected by the outer waves. The interface is partially transparent. To show the two-component electron-hole character of the wave, we use the double lines. The evolution operators U's mix electron-hole components whereas the interface scattering is described by an electronhole scalar $S$-matrix.

(the minus sign in the r.h.s. of Eq. (4.3)). The matrices $U^{(l, r)}$ can be found solving usual quasiclassical equations (see Section B).

In the simplest case of isotropic homogeneous superconductors, the evolution matrices are simply

$$
\begin{aligned}
\mathcal{U}^{(l, r)} & =e^{i \pi+2 i p_{F} a^{(l, r)} / \cos \theta}\left(\frac{1}{2}\left(1+g_{\mathrm{bulk}}^{(l, r)}\right) u^{(l, r)}\right. \\
& \left.+\frac{1}{2}\left(1-g_{\mathrm{bulk}}^{(l, r)}\right) \frac{1}{u^{(l, r)}}\right)
\end{aligned}
$$

where $g_{\text {bulk }}$ stands for the bulk Green's function and

$$
u^{(l, r)}=e^{\frac{2 i \xi(l, r)_{a}(l, r)}{v_{F} \cos \theta}},
$$

$\xi$ is defined by Eq. (2.7) (in the ballistic case, $\xi=$ $\sqrt{\varepsilon^{2}-|\Delta|^{2}}$.

The transformation to the convenient representation Eq. (3.13) where one of the layers (the right one) becomes effectively the normal metal can be performed with the matrix 5

$$
\mathcal{O}=\left(\begin{array}{lr}
1 & a_{\text {bulk }}^{(r)} \\
b_{\text {bulk }}^{(r)} & 1
\end{array}\right)
$$


where $a_{\mathrm{bulk}}^{(r)}$ and $a_{\mathrm{bulk}}^{(r)}$ are the parameters of the MakiSchopohl parametrisation of $g_{\mathrm{bulk}}^{(r)}$.

It follows from the derivation in Sections III and III, that the exact Green's function, e.g. at the point 2 (see Fig. 8), $g_{2}$ can be found using Eq. (2.6b) as Eq. 2.6b),

$$
g_{2}=\frac{1+\mathcal{R}^{(r)} \mathcal{U}^{(r)}}{1-\mathcal{R}^{(r)} \mathcal{U}^{(r)}},
$$

where in accordance with Eq. (3.6) $\mathcal{R}^{(r)}$ can be expressed via the reflection amplitude $\rho$ and the evolution matrix $\mathcal{U}^{(r)}$.

As already mentioned, the exact propagator given by Eq. (3.9) must not be compared with the quasiclassical Green's function because they are different physical objects. Indeed, the Green's function in the Gor'kov equation Eq. (4.1) describes a one-dimensional propagation of a plane wave with definite parallel momentum $\boldsymbol{p}_{\|}$and, therefore, of infinite extension in the direction parallel to the sandwich plane. Being infinite, the multiply reflected waves inevitably overlap and their interference leads to the bound state formation (which would be reveal itself as Fabry-Pérot type resonances if the sandwich was connected to the outside world). On the other hand, a beam-like wave packet built of the plane waves with close values of $\boldsymbol{p}_{\|}$, which behaves like a classical particle in a billiard, does not overlap with itself and the resonances are absent. The wave packet construction, which produces the trajectory picture shown in Fig. 3, is equivalent to the coarse-grained averaging discussed before. The coarse-grained Green's function for the sandwich is given by Eq. (3.17) at the point 2 chosen as a representative point. In what follows we want to show that in an inhomogenious superconductors the interference effects survive the coarse grain averaging. For this, we find the quasiclassical Green's function and compare it with exact coarse-grained solution Eq. 3.17).

\section{A. Quasiclassical Green's function}

In the framework of the quasiclassical theory, an iteration procedure which allows one to find the Green's function of a sandwich, has been suggested ins. Here, we use a modification of the method which is more convenient for the expansion with respect to the path length.

We use the Maki-Schopohl parametrisation of the Green's function 15 ,

$$
\hat{g}=\frac{1}{1-a b}\left(\begin{array}{lr}
1+a b & -2 a \\
2 b & -1-a b
\end{array}\right)
$$

and the boundary conditions for the Andreev amplitudes $a$ and $b^{5,6}, 9$ at the interface.

Consider a collision with the interface, see Fig.9. In accordance with $\mathrm{E}$, the boundary condition can be conveniently expressed via the determinant

$$
\mathcal{D}=\operatorname{det}\left\|1-S \hat{a} S^{\dagger} \hat{b}\right\|
$$

where $S$ is the interface S-matrix Eq. (3.1), and $\hat{a}=$ $\operatorname{diag}\left(a_{1}, a_{2}\right)$ and $\hat{b}=\operatorname{diag}\left(b_{1^{\prime}}, b_{2^{\prime}}\right)$ are diagonal matrices bulit from the "input" Andreev amplitudes at the trajectory points immediately ajacent to the interface (Fig. 8). The determinant reads

$$
\mathcal{D}=R\left(1-a_{1} b_{1^{\prime}}\right)\left(1-a_{2} b_{2^{\prime}}\right)+T\left(1-a_{1} b_{2^{\prime}}\right)\left(1-a_{2} b_{1^{\prime}}\right),
$$

where $R$ and $T$ are the reflection and transmission coefficients. The "output" amplitudes $a_{1^{\prime}, 2^{\prime}}$ and $b_{1,2}$ can be found by the following formulae:

$$
a_{n^{\prime}}=-\frac{\mathcal{D}_{1}^{\left(n^{\prime}\right)}}{\mathcal{D}_{0}^{\left(n^{\prime}\right)}} \quad, \quad b_{l}=-\frac{\mathcal{D}_{1}^{(l)}}{\mathcal{D}_{0}^{(l)}} \quad, \quad n, l=1,2
$$

where we denote

$$
\begin{aligned}
\mathcal{D}_{0}^{(l)} & =\left.\mathcal{D}\right|_{a_{l}=0}, \\
\mathcal{D}_{1}^{(l)} & =\frac{\partial}{\partial a_{l}} \mathcal{D}, \\
\mathcal{D}_{0}^{\left(n^{\prime}\right)} & =\left.\mathcal{D}\right|_{b_{n^{\prime}}=0}, \\
\mathcal{D}_{1}^{\left(n^{\prime}\right)} & =\frac{\partial}{\partial b_{n^{\prime}}} \mathcal{D} .
\end{aligned}
$$

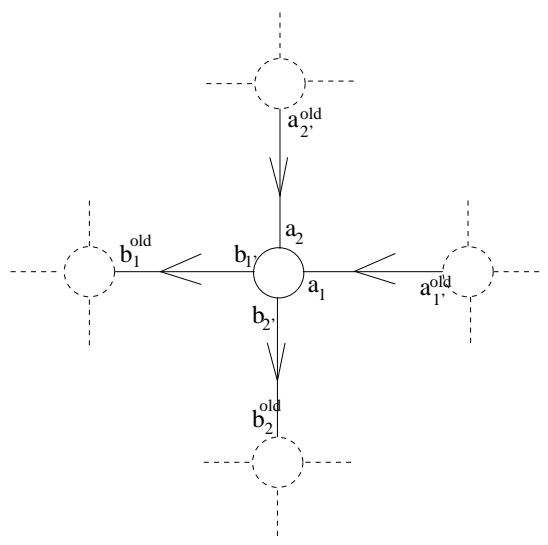

FIG. 9: Scattering on the interface. The incoming channel are 1 and 2, and the outgoing ones are 1' and 2'. The numbering is same as in Fig. 2. The arrows show the direction of the momentum. The input for the interface boundary conditions is the set of Andreev amplitudes $a_{1,2}, b_{1^{\prime}, 2^{\prime}}$. These parameters can be found from the output of the scattering event on the previous collision $a_{1^{\prime}, 2^{\prime}}^{\text {old }}, b_{1,2}^{\text {old }}$.

In the iteration procedure illustrated by Fig. 9, the updated input amplitudes, $a_{1,2}$ and $b_{1^{\prime}, 2^{\prime}}$, are found from the output amplitudes of the previous step, $a_{1^{\prime}, 2^{\prime}}^{\text {old }}$ and $b_{1,2}^{\text {old }}$, from the following relations:

$$
\left(\begin{array}{c}
a_{1,2} \\
1
\end{array}\right) \propto \mathcal{U}^{(l, r)}\left(\begin{array}{c}
a_{1^{\prime}, 2^{\prime}}^{\mathrm{old}} \\
1
\end{array}\right) \quad, \quad\left(\begin{array}{c}
1 \\
b_{1^{\prime}, 2^{\prime}}
\end{array}\right) \propto \mathcal{U}^{(l, r)}\left(\begin{array}{c}
1 \\
b_{1,2}^{\text {old }}
\end{array}\right)
$$

where the "bond" evolution operators $\mathcal{U}^{(l, r)}$ are those in Eq. (4.5). These relation are understood in the following 
sense: For instance, to update $a_{1}$ from the value $a_{1^{\prime}}^{\text {old }}$, one calculates the column $\mathcal{U}^{(l)}\left(\begin{array}{c}a_{1^{\prime}}^{\text {old }} \\ 1\end{array}\right)$, with the evolution matrix Eq. (3.14), and the advanced value $a_{1}$ is found as the ratio of the upper and lower components of the column.

Given the updated input, the new output values are found from Eq. (4.6). The initial input is the bulk value of the parameters. In the following, we use the representation diagonalizing the bulk Green's function in the right layer (as in Section III A). Then the zero step value are $b_{1}=A_{+}, a_{1^{\prime}}=A_{-}, b_{2}=a_{2^{\prime}}=0$, and the evolution operators are those in Eqs. (3.12), and 3.14

Unlike the iteration procedure suggested in 5 , on any iteration steps the parameters $a$ and $b$ have direct physical meaning - they give the Green's function at the central knot of the tree with $4^{n}+1$ knots, $n=0,1, \ldots$ being the number of the iteration steps.

The result of the calculation is the following. In the lowest order with respect to the path length, we recover the exact coarse-grained Green's function discussed in Sect. [IV and derived in Sect. III A. However, the quasiclassical theory reproduces incorrectly terms which are proportional to the product $u^{(r) 2} u^{(l) 2}$, and, of course, higher order terms. Specifically, the quasiclassical theory faithfully reproduces the first terms, $\hat{G}_{q c}$, in the exact expression Eq. (3.17) but it misses the second one, that is $\hat{G}_{n q c}$. We see that $\hat{G}_{n q c}$ in Eq. (3.19) gives the correction to the quasiclassical approximation in the lowest approximation with respect to the path length.

\section{DISCUSSION AND CONCLUSIONS}

In the previous section, we have shown explicitly that the quasiclassical theory gives, strictly speaking, incorrect results when applied to a sandwich with a specular reflecting interface. By this, we confirm some previous observations 11 . We understand this result as due to differences in the actual topology of the trajectories and those assumed in the quasiclassical theory. Our arguments are as follows

In Fig.10, we show fragment of the trajectory space which is large enough to include all the paths contributing to the approximate Green's function in Eq. (3.17): the particle starts in the immediate vicinity of the knot "0" and after travelling around returns back. In the normal metal case, the particle always follows the arrows being the electron, or always moves against the arrows if it is the hole, and there are no return paths. Correspondingly, coarse-grained properties are the same as in the bulk (as it is well-known from the the theory of normal metal films). In a superconductor, the electron-hole conversion is possible due to the Andreev reflection process when the particle hits the interface separating the layers with different order parameters. Then, the particle may move along the arrows as well as in the opposite direction. For instance (see Fig. 10(b)), the particle may move from " 0 " to " 5 " as electron, hit the "5" and being reflected as a hole move back to " 0 " (this give contribution to $u^{(r) 2}$-terms in Eq. (3.18).

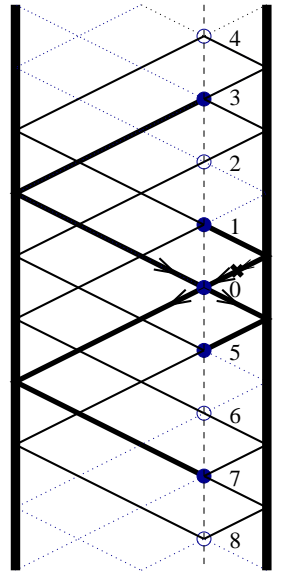

(a)

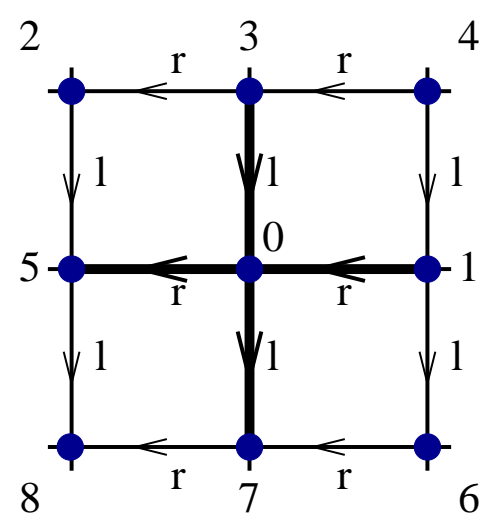

(b)
FIG. 10: Typical trajectory in a sandwich. (a) The cross shows the initial position of the particle near the knot marked by "0". Due to the possibility of the electron-hole conversion, the particle may change its branch and therefore the direction of motion when hitting the interface (dashed line). The connectivity of the trajectory is shown schematically in (b). The knots are numbered as in (a), letters $r$ and $l$ label the pieces of the trajectory in the right or left layer, respectively. This picture refers to the general case when the thickness of the left and right layers are incommensurable. Fig. B(a) shows the case of a symmetric sandwich.

The connectivity in the quasiclassical theory is shown in Fig. 11. The trajectory is tree-like 5 where sequential collisions with the interface are completely uncorrelated as if the surfaces are rough: For instance, the knot $2_{3}$ which can be reached from the knot 3 , is regarded as different from the know $2_{5}$ to which the particle may come from the knot 5 .

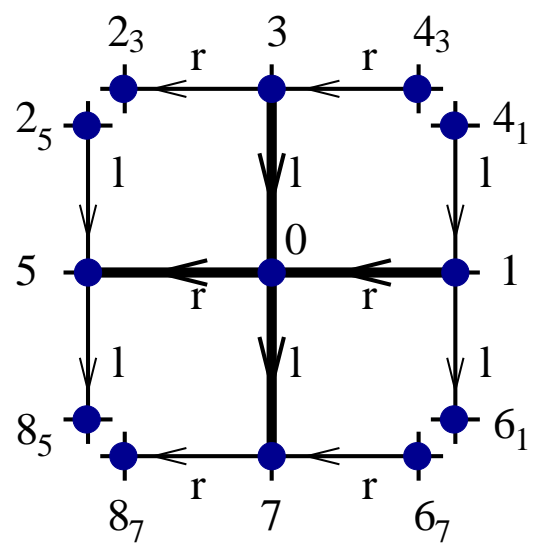

FIG. 11: Tree like trajectory in the quasiclassical theory. Numbering of the notes in the same as in Fig. 10. The subscript shows the knot from which the given knot can be reached.

It is clear from Figs. 10 and 11 that the whole class 
of the paths allowed in the exact theory is not presented in the quasiclassical approach. These are loop-like paths, for instance, " 0 " " $\rightarrow$ " 5 " $\rightarrow$ " 2 " $\rightarrow$ " 3 " $\rightarrow$ " 0 ". This path includes two Andreev reflections - at the knots " 5 " and " 3 ", and its length corresponds to $u^{(l) 2} u^{(r) 2}$. The Fermi momentum phase factors cancel exactly (in a translationally invariant system), and therefore, these paths are immune to the coarse-grain averaging. Summing over all the loop-like paths of this length, one is able to account for the r.h.s. of Eq. (3.19). In the geometry under consideration, the loops are not rare events (as in the weak localization theory), and, nominally, their contribution, i.e. the non-quasiclassical correction, is of order of 1 .

This analysis, which is the main result of the paper, gives the physical explanation of the invalidity of the quasiclassical theory in many layer systems first noticed in Ref.11 and analyzed numerically ind. The terms missing in the quasiclassical theory can be definitely attributed to the loops formed by the interfering paths. Similar conclusion has been made for the case of open geometry calcylating the Josephson current through a layered system 19 . The interference survives coarse-grain averaging because the lengths of the two intefering paths remain equal for any initial condition. In turn, the loops, as closed orbits in the billiard theory, are possible only in very special - integrable - geometries. Here it is important that the sandwich has a perfect in-plane homogeneity and the reflection is specular. Any imperfection on the scale larger than the Fermi wave length (but, perhaps, negligible small compared with the coherence length), transforms the highly connective trajectory in
Fig. 10(a) into the tree in Fig. 11. One may say that the quasiclassical theory is stable relative to small variations of geometry and is applicable in most of the cases. However, there exist physically realizable situations where the conventional quasiclassical theory is inapplicable due to the contribution of the intefering paths. In this case, one should use the modification of the quasiclassical technique suggested in the present paper.

Finally, we want to mention that the physics due to the loops leads to a qualitative effect which is completely beyond the quasiclassical theory. It is well-known that the gauge-invariant potential $\Phi$ does not enter the equation for the quasiclassical stationary retarded Green's function, and therefore, the spectrum found from then Eilenberger equation is completely unaware of its presence. However, the potential $\Phi$ changes the actual value of the Fermi energy at which the Cooper pair condense and, therefore, modifies the value of the Fermi momentum by $\delta p_{F}=\Phi / v_{F}$. Varying $\Phi$, one controls local value $p_{F}$ and the phase acquired by the particle on the intefering paths. Proper choice of conditions, may turn the constructive interference into destructive, and, therefore modify the loop contribution to the density of states. Thus, the loop-like paths provide a physical mechanism for influencing the density of states in a superconductor by the non-equilibrium potential $\Phi$.

\section{Acknowledgments}

This work was supported by the University of Umeå.
* Also at A. F. Ioffe Physico-Technical Institute, 194021 St.Petersburg, Russia.

1 G. Eilenberger, Z. Phys. 214, 195 (1968).

2 A.I. Larkin and Yu.N. Ovchinnikov, Zh. Eksp. Teor. Fiz. 55, 2262 (1968) [Sov. Phys. JETP 28, 1200 (1969)] A.I. Larkin and Yu.N. Ovchinnikov, in Nonequilibrium Superconductivity, ed. D.N. Langenberg and A.I. Larkin, (Elsevier, Amsterdam, 1984).

3 A. Schmid in Nonequilibrium Superconductivity, ed. K.E. Gray (Plenum, N.Y., 1981).

4 J.W. Serene, and D. Rainer, Phys. Rep. 4,221 (1983).

5 A.L. Shelankov and M. Ozana, Phys. Rev. B 61, 7077 (2000).

6 M. Ozana, A. Shelankov, in Proceedings of the Helsinki conference: Symposium on Ultra Low Energy Physics: Methods and Phenomenology, J. Low Temp. Phys., 124, 223-243, (2001).

7 Buchholtz and Rainer Z., Physik B35, 151 (1979)

8 A.V. Zaitsev, Zh. Eksp. Teor. Fiz. 86, 1742 (1984) [Sov. Phys. JETP 59, 1015 (1984)].

9 M. Eschrig, Phys. Rev. B 61, 9061 (2000).

10 M. Ashida, S. Aoyama, J. Hara, and K. Nagai, Phys. Rev. B 40, 8673 (1989); Y. Nagato, K. Nagai and J. Hara, J. Low Temp. Phys. 93, 33 (1993); S. Higashitani and K. Nagai, J. Phys. Soc. Japan. 64, 549 (1995); Y. Nagato and K. Nagai, Phys. Rev. B 51, 16254 (1995); Y. Nagato, M.
Yamamoto and K. Nagai, J. Low. Temp. Phys. 110, 1135 (1998).

11 Y. Nagato, S. Higashitani, K. Yamada and K. Nagai, J. Low. Temp. Phys. 103, 1 (1996); K. Yamada, Y. Nagato, S. Higashitani and K. Nagai, J. Phys. Soc. Jpn. 65, 1540 (1996); K. Nagai, in Quasiclassical theory of superconductivity, ed. D. Rainer and J.A. Sauls, p. 198 (1998).

12 A.L. Shelankov, J. Low Temp. Phys. 60, 29 (1985).

13 A.L. Shelankov, Zh. Eksp. Teor. Fiz. 78, 2359 (1980), Sov. Phys. JETP 51, 1186 (1980).

14 A.F. Andreev, Zh. Eksp. Teor. Fiz. 46, 1823 (1964) [Sov. Phys. JETP 19, 1288 (1964)].

15 N. Schopohl, and K. Maki, Phys. Rev. B 52, 490 (1995); N. Schopohl "Transformation of the Eilenberger Equations of Superconductivity to a Scalar Riccati Equation", condmat/9804064 and in Quasiclassical theory of superconductivity, ed. D. Rainer and J.A. Sauls, p. 88 (1998).

16 The identification of "+" ("-") with the electron (hole), is the opposite when one analyses the advanced Green's function $\hat{g}^{A}$.

17 A.L. Shelankov, Pis'ma Zh. Eksp. Teor. Fiz. 32, 122 (1980) [JETP Lett. 32, 111 (1980)].

18 For the potential $V(x)=\frac{\lambda}{m} \delta(x)$, the scattering amplitudes $\operatorname{read} r=-i \tilde{\lambda} /(1+i \tilde{\lambda}) \quad, \quad t=1 /(1+i \tilde{\lambda}) \quad, \quad \tilde{\lambda}=\lambda / p_{F_{x}}$

19 M. Ozana, A. Shelankov, J. Tobiska, in preparation. 


\section{APPENDIX A: OBSERVABLES}

For calculating observables the Green's function at coinciding arguments $G(x, x)$ is needed. It follows directly from the continuity of $G\left(x, x^{\prime}\right)$ for $x=x^{\prime}$ and the definition of $\hat{g}^{( \pm)}$that

$$
\begin{aligned}
\hat{G}(x, x) & =\frac{1}{2}(\hat{G}(+x, x)+\hat{G}(-x, x))= \\
& =\frac{1}{2}\left(\hat{G}^{(+)}(+x, x)+\hat{G}^{(-)}(+x, x)\right. \\
& =\frac{1}{2}\left(\hat{g}^{(+)}(x)+\hat{g}^{(-)}(x)\right) .
\end{aligned}
$$$$
=\frac{1}{2}\left(\hat{G}^{(+)}(+x, x)+\hat{G}^{(-)}(+x, x)+\hat{G}^{(+)}(-x, x)+\hat{G}_{\text {calculation }}^{(-T)}\right. \text { the purpe of this section is to present a method for }
$$

The local density of states can be written in terms of $\hat{\mathbb{G}}$ as follows

$$
\nu(x)=\frac{i}{2 \pi} \lim _{x \rightarrow x^{\prime}} \operatorname{Tr}\left(\mathbb{G}\left(x, x^{\prime}\right)-\mathbb{G}^{A}\left(x, x^{\prime}\right)\right) .
$$

From here the following expression for LDOS via the 1point Green's function $g^{( \pm)}(x)$ can be found

$$
\nu(x)=\frac{1}{2 \pi v_{F}} \Re \operatorname{Tr}\left[\left(\hat{g}^{(+)}(x)+\hat{g}^{(-)}(x)\right) \hat{\tau}_{z}\right]
$$

This is the formula for the local density of states at a point $x$ expressed in terms of the 1-point Green's function $g(x)$.

The standard quantum mechanical expression for the current density via Gor'kov Green's function reads

$$
j(x)=\frac{1}{4 m} \lim _{x \rightarrow x^{\prime}}\left(\frac{\partial}{\partial x}-\frac{\partial}{\partial x^{\prime}}\right) \operatorname{Tr} \hat{\mathbb{G}}^{K}\left(x, x^{\prime}\right) .
$$

In the equilibrium the Keldysh Green's function can be expressed as $\hat{\mathbb{G}}^{K}=\left(\hat{\mathbb{G}}-\hat{\mathbb{G}}^{A}\right) \tanh \varepsilon / 2 T$. The differentiation with respect to $x$ and $x^{\prime}$ is performed using identities $\partial_{x} \hat{G}^{( \pm)}= \pm i p_{G} \hat{G}^{( \pm)}$and $\partial_{x^{\prime}} \hat{G}^{( \pm)}=\mp i p_{G} \hat{G}^{( \pm)}$. This leads to

$$
j(x)=-v_{F} \tanh \frac{\varepsilon}{2 T} \lim _{x \rightarrow x^{\prime}} \Im \frac{1}{i v_{F}} \operatorname{Tr}\left(\hat{G}^{(+)}-\hat{G}^{(-)}\right) \tau_{z} .
$$

Finally we take the limits $x \rightarrow x^{\prime}$ and get the expression for the current density in terms of 1-point Green's function $\hat{g}(x)$

$$
j(x)=\frac{1}{2} \tanh \frac{\varepsilon}{2 T} \Re \operatorname{Tr}\left[\left(\hat{g}^{(+)}(x)-\hat{g}^{(-)}(x)\right) \hat{\tau}_{z}\right] .
$$

\section{APPENDIX B: EVOLUTION MATRIX}

One can always present $U\left(x, x^{\prime}\right)$ in the following form

$U\left(x, x^{\prime}\right)=a\left(x, x^{\prime}\right)\left(\begin{array}{c}1 \\ \alpha\left(x, x^{\prime}\right)\end{array}\right) \otimes(1,0)+b\left(x, x^{\prime}\right)\left(\begin{array}{c}\beta\left(x, x^{\prime}\right) \\ 1\end{array}\right) \otimes($

where

$$
a(x, x)=b(x, x)=1 \quad, \quad \alpha(x, x)=\beta(x, x)=0
$$

as required by the condition $U(x, x)=1$. Since the evolution matrix must generate a solution for arbitrary initial condition at $x^{\prime}$, the two terms in Eq. (B1) must satisfy the quasiclassical equation separately.

From Eq. (2.3). the two-component "wave function" $\left(\begin{array}{l}u \\ v\end{array}\right)=\left(\begin{array}{c}a \\ a \alpha\end{array}\right)$ and $=\left(\begin{array}{c}b \beta \\ b\end{array}\right)$ considered as functions of $x$, satisfy the Andreev-type linear first-order differential equation. It is known from the literature 11.15 that the ratio $u / v$ obeys the nonlinear Riccati equation,

$i \frac{\partial}{\partial x} \alpha=2 \varepsilon \alpha+\Delta^{* R}+\Delta \alpha^{2} \quad, \quad-i \frac{\partial}{\partial x} \beta=2 \varepsilon \beta+\Delta^{* R} \beta^{2}+\Delta$.

with the initial condition in Eq. (B2).

Known $\alpha$ and $\beta$, parameters $a\left(x, x^{\prime}\right)$ and $b\left(x, x^{\prime}\right)$ can be found as

$$
a\left(x, x^{\prime}\right)=e^{i \int_{x^{\prime}}^{x} d x(\varepsilon+\Delta \alpha)} \quad, \quad b\left(x, x^{\prime}\right)=e^{-i \int_{x^{\prime}}^{x} d x\left(\varepsilon+\Delta^{* R} \beta\right)}
$$

This completes the derivation of the evolution matrix in a general case. 